\title{
L'éducation au territoire au sein du château de Bouthéon (Loire)
}

\section{Olivier Rousseau}

\section{(2) OpenEdition \\ Journals}

Édition électronique

URL : https://journals.openedition.org/ere/3432

DOI : $10.4000 /$ ere.3432

ISSN : 2561-2271

Éditeur

Centr'ERE

Référence électronique

Olivier Rousseau, "L'éducation au territoire au sein du château de Bouthéon (Loire) », Éducation relative à l'environnement [En ligne], Volume 15 - 1 | 2019, mis en ligne le 23 septembre 2019, consulté le 16 octobre 2021. URL : http://journals.openedition.org/ere/3432 ; DOI : https://doi.org/10.4000/ere.3432

Ce document a été généré automatiquement le 16 octobre 2021.

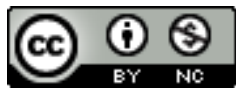

La revue Éducation relative à l'environnement est mise à disposition selon les termes de la Licence Creative Commons Attribution - Pas d'Utilisation Commerciale 4.0 International. 


\title{
L'éducation au territoire au sein $\mathrm{du}$ château de Bouthéon (Loire)
}

\author{
Olivier Rousseau
}

1 A l'échelle de la dimension stratégique du processus muséal ${ }^{1}$, la politique de mise en valeur du château de Bouthéon, établissement patrimonial que nous dirigeons depuis une dizaine d'années, s'inscrit dans une double logique de développement local (Rousseau, 2013). En effet, cette politique vise à considérer ce musée-monument comme une ressource pour la commune-gestionnaire permettant, d'une part, de générer des retombées économiques via la fréquentation induite, et, d'autre part, d'agir directement sur le territoire communal, par exemple, par la production de légumes bio pour les cantines scolaires ${ }^{2}$ ou par le ramassage hebdomadaire des poubelles dans la zone de loisirs des bords de Loire avec notre cheval de trait auxois de « collection $»^{3}$. Au niveau du projet muséographique, cette finalité développementaliste se retrouve également au niveau des intentions de départ de la collectivité locale puisqu'il s'agissait de faire de Bouthéon une "porte d'entrée », un lieu promettant une compréhension du monument (le château) et d'un territoire donné (le Forez), afin de concentrer un flux de fréquentation sur ce site particulier puis de le rediffuser sur ce territoire en ayant invité le visiteur à le parcourir et l'apprécier, fort des connaissances acquises sur celuici.

2 Nous souhaitons ici montrer comment, à l'échelle du processus patrimonial et médiatique, notre établissement peut s'analyser, également, comme un musée d'éducation au territoire contribuant à l'appropriation du Forez par ses habitants et ses visiteurs, dans une double perspective de référence identitaire et de développement local.

3 Afin de porter ce regard réflexif sur notre offre muséale, nous allons mobiliser la typologie de Girault et Barthes (2016). Ces auteurs proposent une structuration du champ de l'éducation aux territoires, selon trois significations possibles de ce dernier terme. En effet, ils rappellent qu'en épistémologie de la géographie, le territoire peut être «lieu de vie", «lieu de pouvoir » ou « lieu des rapports d'appropriation d'une société à son espace géographique ». Ils ajoutent que deux notions importantes doivent 
également être prises en compte au niveau des finalités éducatives: l'identité et la ressource. Enfin, ces auteurs précisent comment les différents courants éducatifs en lien avec les territoires s'intègrent avec la typologie proposée, avec des objectifs et des valeurs parfois opposés. Partant de cette grille d'analyse, nous nous focaliserons sur deux approches identifiables au château de Bouthéon. D'une part, l'interprétation du lieu de vie dans un but identitaire et, d'autre part, l'éducation au développement local durable.

\section{Contexte et projet muséal du château de Bouthéon}

4 Andrézieux-Bouthéon est une ville française de près de 10000 habitants ${ }^{4}$ située sur la rive droite du fleuve Loire, au sud de la plaine du Forez ${ }^{5}$, en périphérie de la métropole de Saint-Étienne et de l'aire métropolitaine de Lyon (région Auvergne-Rhône-Alpes). La commune se compose, schématiquement, d'un tiers de zones naturelles et agricoles, d'un tiers de zones d'habitations et d'un tiers de zones industrielles, artisanales et commerciales. Son emplacement géographique, son activité économique (plus de 10000 emplois, occupés à $87 \%$ par des habitants d'autres communes), et son niveau d'équipements structurants et de services (lycées, théâtre, palais des sports, centre nautique, aéroport, plus de 200 associations, etc.) font d'Andrézieux-Bouthéon un des pôles d'attractivité majeurs du département. Intégré à cette dynamique, le domaine du château de Bouthéon, propriété de la commune depuis 1995 et ouvert à la visite depuis 2007, est devenu rapidement un des lieux les plus fréquentés du département avec 70 à 80000 entrées par an.

5 La première mention du château de Bouthéon remonte à $1224^{6}$ où une charte évoque un castrum de Boteon dépendant du comte du Forez. Au fil des siècles, ce château vit passer plusieurs familles dont les La Fayette (XVe siècle), les Bourbon (XVe-XVIe siècles) et les Gadagne (XVIe-XVIIIe siècles). Ces derniers transformeront définitivement l'ancienne place forte dominant le fleuve Loire et son domaine agricole en une résidence Renaissance dédiée aux arts et aux lettres, avant de la laisser progressivement se dégrader, faute de moyens financiers pour l'entretenir. À la fin du XIXe siècle, un rubanier stéphanois, Claude Coignet (1820-1911), restaure l'édifice qui traversera ensuite le XXe siècle en devenant notamment un lieu d'accueil pour des soldats blessés en 1914-1915, puis pour des réfugiés civils des deux guerres mondiales, et enfin pour des colonies de vacances dans les années 1950. Redevenue habitation bourgeoise au cours des années 1960 et 1970, une partie de la propriété est aménagée par Paul Grousset (1912-1978), un industriel local, par ailleurs membre de la Société protectrice des animaux, pour sauvegarder des équidés et des oiseaux. Ce court rappel historique permet d'esquisser l'«esprit des lieux $»^{7}$ : un château dont l'identité est étroitement liée à l'histoire de son territoire et de ses habitants.

6 Aujourd'hui, nous définissons le château de Bouthéon comme un musée de territoire dédié à la construction identitaire et au développement du Forez. De par les fonctions muséales de présentation et d'animation mais également du fait des attentes diverses des utilisateurs (Gob et Drouet, 2004) ${ }^{8}$, le château de Bouthéon propose différents outils complémentaires d'éducation au territoire. L'approche se veut globale et s'appuie sur les différentes dimensions du visiteur : affective, gustative, sociale, physique, cognitive, etc. 
7 Afin de satisfaire à ce projet muséal, les missions du château de Bouthéon se déclinent en plusieurs dispositifs, activités ou actions. Pour apporter deux éclairages différents sur les rapports possibles entre cette institution muséale et l'éducation aux territoires, nous avons retenu, au sein de notre offre variée, le media exposition permanente et l'un de nos évènementiels.

\section{Une triple interprétation du lieu de vie par le media exposition}

Selon Girault et Barthes (2016), divers courants d'interprétation s'inscrivent dans une perspective d'éducation au territoire en tant que lieu de vie et dans une finalité identitaire, l'appropriation du territoire passant alors par un processus mental collectif d'adhésion à des valeurs et à un ensemble de caractéristiques spécifiques de ce territoire. Nous pouvons le vérifier dans le cas de notre établissement, alors même que cet objectif d'appropriation patrimoniale du territoire était absent du projet muséographique initial.

9 En effet, la fonction muséale de présentation permanente se matérialise, d'une part, dans le château, par un centre d'interprétation du territoire forézien et par un aquarium consacré au fleuve qui coule à ses pieds et, d'autre part, à l'emplacement des anciens jardins dudit château, par un parc animalier et botanique. De ce fait, chacun de ces espaces propose une approche interprétative du territoire (lieu de vie) que nous pouvons relier au concept d'expérience patrimoniale forgé par Viel (2008), c'est-à-dire une expérience de sens (esprit du lieu), de connaissance (savoirs) et de conscience (réflexion) s'appuyant à la fois sur le château et son territoire. Ainsi, ces trois espaces participent à la construction et la reconnaissance d'une identité forézienne tant pour les habitants que pour les visiteurs de passage.

\section{Dans ce château du Forez, l'expérience globale d'un territoire}

Le Centre d'interprétation du Forez s'étend sur une grande partie du château et combine dispositifs audiovisuels, maquettes, espaces d'immersion et collection d'objets liés, entre autres, à la vie industrielle et agricole. Sont notamment présentés, dans une perspective historique, géographique et ethnologique et sous l'angle des particularités locales, des paysages et milieux, des architectures remarquables, des productions et savoir-faire. Par exemple : étangs de la plaine, landes des monts, constructions en terre crue, prieurés, produits remarquables (Appellation d'Origine Contrôlée côtes-du-Forez et Appellation d'Origine Protégée fourme de Montbrison, eau minérale gazeuse Badoit, boules de pétanque Obut), industrie des tresses, lacets et rubans ou première ligne de chemin de fer en France (acheminant, dès 1827, le charbon stéphanois jusqu'au port d'Andrézieux).

11 Plusieurs thématiques sont ainsi mises en valeur afin d'offrir aux visiteurs, au cours de leur visite du château, une expérience muséale et forézienne globale tout en les invitant à parcourir physiquement le territoire. Relevons que dans ce centre d'interprétation, le ressort ludique est souvent utilisé, par exemple dans l'ancienne cuisine où un jeu invite à identifier les principaux ingrédients de recettes locales (la 
râpée, le patia et le pissou), avec, en récompense, la possibilité de repartir avec la ficherecette complète.

Par ce parcours scénographié dans le château, nous proposons donc une expérience patrimoniale autour des éléments constituants, saillants voire différenciateurs, dans le temps et l'espace, du Forez.

\section{Dans ce château de la Loire : l'éco-socio-système d'un fleuve}

Dans l'Aquarium du château de Bouthéon (également dénommé Centre d'interprétation du fleuve Loire), par la voix d'une comédienne et via des dispositifs sonores, la Loire commente son parcours et ses rencontres, des sources jusqu'à l'océan. Elle met en perspective ses liens complexes avec notre société (paysages, loisirs, navigation, crues, alimentation, pollutions, etc.) à travers cinq milieux scénographiés (les sources, les gorges, les plaines, les villes, l'estuaire) et 36 espèces de poissons vivants dans un volume total d'eau de 60000 litres.

Cette présentation ne se focalise donc pas sur le territoire du Forez mais sur le fleuve qui le traverse et relie différents milieux de vie et populations, formant ainsi un immense territoire (lieu de vie) où se construit une identité ligérienne qui s'intègre aux identités plus locales.

15 L'intérêt d'une telle présentation muséale tient, notamment, dans la possibilité d'appréhender, de manière globale et systémique, sur seulement $250 \mathrm{~m}^{2}$, les réalités environnementales d'un fleuve long de $1006 \mathrm{~km}$. La Loire peut alors être envisagée comme un "éco-socio-système ", selon l'expression de Louis Goffin (1998), soit un système d'interactions, dans le temps et l'espace, entre des éléments naturels ou construits et des éléments sociétaux. Notons que cette définition se rapproche de celle du territoire perçu comme le lieu des rapports d'appropriation entre une société et son espace (Girault et Barthes, 2016). En effet, l'Aquarium ne présente pas le fleuve uniquement comme un lieu identitaire, mais également comme un lieu aux multiples valeurs d'usage et de non-usage (patrimoine, biodiversité, loisirs, production, aménagement, etc.).

Par ailleurs, cette expérience patrimoniale autour de la Loire permet de pointer la complexité des problématiques environnementales dont les enjeux sont rarement circonscrits à une échelle locale. Par exemple, la problématique des barrages sur la Loire, dont deux des trois grands ouvrages encadrent la plaine du Forez, est mise en scène et abordée dans cet Aquarium: production électrique, écrêtage des crues, maintien du niveau d'eau (refroidissement des centrales nucléaires et consommation humaine), migration des anguilles et saumons rendue impossible, création de bases nautiques sur les retenues d'eau, bouleversement des écosystèmes, concentration des sédiments et des polluants, etc. Il n'est donc pas étonnant que cet espace soit également utilisé dans une finalité éducative de gestion durable des ressources fluviales ${ }^{9}$.

\section{Dans les jardins du château : la biodiversité domestique}

Le dernier espace d'exposition permanente du lieu de vie est le parc du château de Bouthéon qui a connu, au fil des siècles, divers usages. Aujourd'hui, ce parc présente et sauvegarde des animaux d'élevage liés à des terroirs spécifiques tels que la poule cou$n u$ du Forez ${ }^{10}$, la chèvre du Massif central ou le mouton mérinos d'Arles. Des animaux à 
fonction ornementale sont également élevés comme le canard coureur indien ou la carpe koï. Les races présentées, une quarantaine, ne sont donc pas originaires uniquement du Forez. Il s'agit plutôt de montrer quelques témoins actuels du travail de sélection pratiquée par les humains sur la nature. Une sélection artificielle, culturelle, différenciée suivant les territoires et les finalités (critères esthétiques, production alimentaire ou non-alimentaire, fourniture d'un travail). Grâce à des panneaux d'interprétation, des vidéos et des animations, cet espace permet d'interroger la disparition de cette biodiversité domestique, synonyme de perte d'identité territoriale, au profit d'une standardisation industrielle.

Selon une logique similaire, le parc propose de découvrir également plusieurs environnements horticoles : roseraie, jardin à la française, arboretum, vergers, potager, etc. Outre leur vocation d'agrément ou de production, ces environnements rappellent que la plupart des espèces cultivées actuellement en Europe n'ont pas été domestiquées sur ce continent mais au Moyen-Orient, en Asie ou aux Amériques. De ce fait, nos territoires ne comportent pas que des éléments identitaires, au sens de différenciateurs d'un territoire à un autre. Ils incluent également des éléments exogènes et que notre société, devenue mondialisée, s'est appropriée, ce qui souligne l'aspect dynamique des pratiques de patrimonialisation.

In fine, de cette expérience patrimoniale liée au contact direct et sensible avec l'environnement créé dans le parc, où les interactions entre le naturel et le culturel sont mises en avant, peut émerger chez les visiteurs un questionnement autour du "bon usage » de la nature puisque, dans nos lieux de vie, "nous ne rencontrons plus que des objets hybrides, (mixtes de nature et de culture)" (Larrère et Larrère, 1997). Ainsi, comme pour l'Aquarium, ce traitement muséal de l'identité territoriale peut permettre aussi l'émergence d'une réflexion plus large.

\section{L'éducation au développement durable par la manifestation Couleur Nature, l'environnement en jeux}

Le territoire pouvant être défini comme le lieu des rapports d'appropriation entre une société et son espace, Girault et Barthes (2016) rappellent que cette appropriation, tout en pouvant être fondatrice d'identité, peut être également reliée à la notion de ressources territoriales structurant l'existence pratique des individus. Dans ce cadre, l'éducation au territoire (comme lieu des rapports sociaux) peut renvoyer, dans une perspective ressourciste, aux courants de l'éducation au développement durable, en particulier ceux accompagnant les politiques publiques locales basées sur l'exemplarité et la reproductibilité des actions.

\section{Une manifestation issue de l'Agenda 21 de la ville}

Depuis 2010, Andrézieux-Bouthéon est engagée dans une démarche d'Agenda 21, formalisée par un programme d'actions qui a reçu, en 2013, le label national, Agenda 21 local France. À travers les 18 thématiques structurant le document de synthèse, le château de Bouthéon est pris souvent en exemple, en particulier dans les aspects relatifs à la pédagogie dont les objectifs sont d'« informer, sensibiliser et associer l'ensemble des habitants sur les questions de développement durable ». En effet, le site accueille une manifestation spécifiquement créée par la collectivité pour répondre à la 
demande d'informations formulée par les habitants au cours des réunions thématiques issues de la démarche d'Agenda 21 : Couleur Nature, l'environnement en jeux.

Les objectifs assignés à cet évènement annuel sont de :

- rassembler, le temps d'un weekend, des structures qui œuvrent pour un développement durable ;

- valoriser leurs actions ;

- présenter ces initiatives au public ;

- sensibiliser le plus grand nombre aux enjeux d'un développement durable par un temps festif et ludique.

Compte tenu de la cohérence avec son projet muséal et sa mission d'animation d'une part, et d'autre part de la volonté de ne pas toucher uniquement les habitants de la commune mais de rayonner plus largement, le parc du château de Bouthéon fut logiquement désigné comme lieu d'accueil. Cette manifestation pouvait, de ce fait, s'appuyer sur un modèle d'organisation et sur des moyens humains et matériels habituellement mobilisés sur ce site par la commune pour d'autres grands évènements ${ }^{11}$. Elle bénéficiait également d'un lieu attractif venant renforcer l'attrait de cette opération. Par cet évènementiel, le château de Bouthéon avait, en retour, l'opportunité de renforcer son offre et la fréquentation de son site.

\section{Couleur Nature, l'environnement en jeux : le jeu sert-il l'enjeu?}

Dès la première année, en 2011, plus de 5000 visiteurs sont venus découvrir, au fil des allées du parc, des acteurs locaux de l'environnement et leurs espaces d'information et d'animation pour préserver la ressource en eau, être solidaire, consommer de manière responsable, maitriser les énergies, favoriser la biodiversité ou produire dans le respect de la nature. L'éventail des structures présentes était large : associations de protection de la nature, d'éducation à l'environnement, d'éco-consommation, acteurs de l'économie sociale et solidaire, fédération de chasse et de pêche, collectivités locales, sociétés de gestion de l'eau et de l'énergie, etc. Afin d'attirer les familles, ces stands étaient complétés par diverses animations : vols captifs en montgolfière, exposition interactive sur le changement climatique, grimpe d'arbres encadrée, pêche à la truite, balades en calèche, manège à vélo, jeux en bois, etc.

Huit ans après, cette manifestation de plein air attire toujours autant : en moyenne plus de 5000 entrées sont toujours comptabilisées, avec une pointe à plus de 8000 en 2015 et 2018. Une cinquantaine de stands et d'activités sont désormais proposés et une opération nationale est intégrée au dispositif : le Kids FitDays MGEN ${ }^{12}$. Il s'agit d'un tour de France à étapes conjuguant sport, bien-être, engagement et solidarité. Il comprend un triathlon dédié aux enfants et des ateliers pédagogiques : citoyenneté et droits de l'enfant, sapeurs-pompiers, nutrition, sécurité routière, déchets, gestes et postures, etc.

Couleur Nature est pilotée, depuis l'origine, par M. Eric Vocanson, deuxième adjoint au Maire, en charge du développement durable, de l'environnement et des grands évènements. Voici son analyse de cette animation:

Nous voulions proposer une manifestation instructive sur le développement durable. Pour y parvenir, celle-ci devait être ludique afin de sensibiliser un large public et favoriser l'attention des visiteurs. Il faut impérativement que cet aspect ludique soit présent sur la plupart des stands car nous sommes sans cesse abreuvés d'informations sur cette thématique et devons donc faire attention à ne pas créer 
une saturation auprès du public. De plus, l'initiation au développement durable - tri des déchets, etc. - passe inévitablement par les enfants et ceux-ci veulent d'abord se divertir.

Nos objectifs sont atteints : chaque année, nous avons une diversité d'acteurs du développement durable et le public est composé, principalement, de familles avec des enfants de tous âges! Les visiteurs viennent sur cet événement pour découvrir, s'initier, s'amuser, contrairement, par exemple, à notre fête de la courge où ils viennent pour consommer et retrouver les mêmes produits chaque année. Pour Couleur Nature, il faut donc savoir se renouveler afin de proposer régulièrement des animations nouvelles, tout en gardant certaines valeurs sûres.

\section{et sa thématique, certains acteurs interrogés, entre autres des représentants} d'associations engagées pour un développement durable, regrettent que l'aspect « fête familiale » semble parfois prendre le dessus sur leur propre objectif d'éducation à la citoyenneté, notamment autour de certaines questions socialement vives telles que la transition énergétique ou les changements climatiques : «beaucoup viennent surtout consommer des animations ", "le raisonnement qui consiste à sensibiliser les parents par le biais des enfants trouve de vraies limites [...], on se pose d'ailleurs la question de renouveler notre participation", "des familles ne prennent pas toujours le temps d'échanger avec nous ", "sur certains stands, le lien avec le développement durable n'est pas toujours explicite", "promouvoir des bons gestes à adopter et proposer des activités pour les enfants c'est bien, mais est-ce vraiment adapté à la complexité des problématiques environnementales?».

Cependant, cette manifestation a d'abord vocation à familiariser, en un lieu dédié et sur le temps du loisir familial, un public néophyte aux problématiques, actions et acteurs possibles en matière de développement local durable. Elle s'inscrit donc dans une démarche informative, behavoriste et positiviste, consistant à apporter des connaissances, à montrer l'exemple, dans un contexte ludique et dans l'optique d'un changement de comportement. Malheureusement, elle n'inclut pas de réflexion méthodologique ou didactique permettant d'évaluer ses effets en dehors du niveau de fréquentation. Ce n'est qu'un outil éducatif parmi d'autres, visant une adhésion du citoyen et non le développement de sa pensée critique, objectif dont la mise en œuvre nous semble peu compatible avec un dispositif de type évènementiel grand public.

\section{Conclusion}

En 1607, Honoré d'Urfé introduisait son roman l'Astrée en ces termes: "Auprès de l'ancienne ville de Lyon, du côté du soleil couchant, il y a un pays nommé Forez, qui en sa petitesse contient ce qu'il y a de plus rare au reste des Gaules ». Comme le Forez de Céladon, le musée de territoire est un lieu d'histoire(s) et de rencontres, un microcosme codifié, parfois idéalisé. Mais contrairement à l'utopie d'un équilibre parfait entre nature et culture tel que représenté dans le roman pastoral, le musée de territoire, en tant qu'institution permanente, au service de la société et de son développement, se doit d'éduquer à la complexité de son environnement et favoriser l'appropriation territoriale dans une perspective à la fois identitaire et de développement local.

Comme nous avons pu le montrer, le Château de Bouthéon s'inscrit clairement dans cette dynamique d'éducation aux territoires. Toutefois, nous avons pu relever aussi quelques limites dans l'utilisation de la typologie de référence de Girault et Barthes. En 
effet, celle-ci ne permet pas d'appréhender toutes les ambivalences de certaines approches. Il en est ainsi, notamment, du centre d'interprétation du fleuve Loire pouvant facilement basculer de la finalité éducative identitaire à celle de la ressource à gérer (sans que cela ne soit pour autant une contrainte), ou encore de la manifestation sur le développement durable qui est un succès en termes d'objectifs initiaux mais qui présente au moins deux risques inhérents à ce type d'action. D'une part, la dimension festive pourrait devenir une finalité et non un moyen, et, d'autre part, certains acteurs du développement durable pourraient ne plus s'investir dans la manifestation car un tel dispositif laisse peu de place à la construction d'une expression citoyenne et à une réflexion sur des problématiques ne relevant pas uniquement de comportements individuels.

31 Il n'en demeure pas moins que la typologie utilisée nous a permis de prendre du recul et clarifier notre offre et ses potentialités. Elle nourrira notre réflexion sur la gestion $\mathrm{du}$ projet muséal au niveau du développement et du renouvellement de nos présentations et actions. En particulier, nous porterons notre attention sur les courants de l'éducation relative à l'environnement et au territoire que nous n'avons pas identifié dans notre structure, comme ceux liés à la critique sociale ou centrés sur la communauté.

\section{BIBLIOGRAPHIE}

Giraut Y. et Barthes A. (2016). Postures épistémologiques et cadres théoriques des principaux courants de l'éducation aux territoires. Éducation relative à l'Environnement : Regards - Recherches Réflexions, 13 (2).

Gob, A. et Drouguet, N. (2004). La muséologie - Histoire, développements, enjeux actuels. Paris : Armand Colin éditeur.

Goffin, L. (1998). L'environnement comme éco-sociosystème. In M. Loriaux (dir.), Populations et développements : une approche globale et systémique. Louvain-la-Neuve et Paris : Academia-Bruylant et L'Harmattan, p. 199-230.

Larrère, C et Larrère, R. (1997). Du bon usage de la nature. Pour une philosophie de l'environnement. Paris : Aubier.

Prats, M. et Thibault, J.-P. (2003). Qu'est-ce que l'esprit des lieux ? Dans $14^{\text {th }}$ ICOMOS General Assembly and Scientific Symposium : «Place, Memory, Meaning:Preserving Intangible Values in Monuments and Sites ", 27-31 oct 2003, Victoria Falls, Zimbabwe. En ligne : https://www.icomos.org/ victoriafalls2003/papers_fre.htm

Rousseau, O. (2005). Analyse de la genèse d'un dispositif muséal à vocation touristique : Nocturnia et le Pôle Nature de l'Argonne ardennaise. Thèse de doctorat, Muséum national d'Histoire naturelle.

Rousseau, O. (2013). Le château de Bouthéon : un lieu patrimonial dédié au développement local. Dans Y. Girault et M. Van Praët (dir.). MuséoMuséum, 20 ans d'enseignement de la muséologie au Muséum national d'Histoire naturelle: Actes du colloque des 12 et 13 décembre 2012 (p. 107-117). Dijon/ Paris : OCIM/MNHN. 
Viel, A. (2008). Quand souffle l'« esprit des lieux ». Dans 16th ICOMOS General Assembly and International Symposium : "Finding the spirit of place - between the tangible and the intangible ", 29 sept - 4 oct 2008, Quebec, Canada. En ligne : http://openarchive.icomos.org/164

\section{NOTES DE FIN}

1. Au sein du processus muséal, superposée aux dimensions patrimoniale et médiatique, la dimension stratégique se focalise sur la recherche, pour un territoire donné, de répercussions positives issues de la présence d'un musée, comme par exemple le développement touristique (Rousseau, 2005).

2. En 2018, près d'un hectare de terres dans et aux abords du château sont cultivés pour fournir la cuisine centrale de la ville en légumes certifiés bio. Cette production en interne fait l'objet d'actions de valorisation culturelle et pédagogique.

3. Présenté et conservé au sein du parc animalier, l'auxois est l'une des races de chevaux de trait français les plus menacées de disparition.

4. Les données démographiques et économiques de cette section sont issues du Plan Local d'Urbanisme de 2013 ainsi que du dossier INSEE pour l'année 2015 de la commune d'AndrézieuxBouthéon.

5. Le Forez est une région naturelle et historique française correspondant, globalement, à la partie centrale de l'actuel département de la Loire. Constituée d'une vaste plaine traversée par le fleuve et entourée de moyennes montagnes, il offre un paysage rural qui s'est périurbanisé dans sa partie sud, intégrée à l'aire urbaine de Saint-Etienne (515.000 habitants).

6. Dans cet article, les données factuelles sur l'histoire et l'offre muséale du château de Bouthéon proviennent des diverses sources documentaires produites et/ou conservées dans l'établissement.

7. L'esprit des lieux est un concept qui peut se définir comme une «synthèse des différents éléments, matériels et immatériels, qui contribuent à l'identité d'un site " (Prats et Thibault, 2013). Pour Viel (2008), au cœur d'un lieu marqué par le temps et les hommes, c'est «une émotion, un aura, un esprit qui transcende le présent, défie les modes d'intervention, interpelle les mémoires et oblige à pénétrer l'univers de la complexité ".

8. Gob et Drouet distinguent quatre fonctions ou missions assignées au musée (présenter, conserver, rechercher et animer) et six types d'attentes de ses usagers (loisir et plaisir, découverte, mémoire, marqueur culturel, activité touristique, rituel).

9. Dans le cadre d'activités éducatives spécifiques, l'Aquarium permet de faire comprendre comment un évènement localisé peut avoir un impact plus global. L'un des exemples utilisé est le barrage hydroélectrique de Grangent, situé à $8 \mathrm{~km}$ du château de Bouthéon. En 1967, sa vidange totale, pour l'entretien décennal des vannes d'évacuation, avait libéré des boues toxiques (issues de l'activité humaine en amont) et entraîné une pollution du fleuve sur 200 à $400 \mathrm{~km}$ en aval, avec des conséquences sur la vie aquatique, l'agriculture ou encore la potabilité de l'eau. Depuis, le process de maintenance a évolué pour éviter la libération des boues. Cependant, celles-ci continuent de s'accumuler en amont du barrage, favorisant artificiellement, au niveau de la base nautique, le développement de cyanobactéries dont certaines espèces produisant des toxines.

10. La race de poule cou-nu du Forez a été créée dans les années 1950 par Henri Calemard, descendant de la famille Coignet qui fut propriétaire du château de Bouthéon de 1871 à 1938. 
11. Le parc accueille différentes manifestations dont la Fête de la Courge et des saveurs d'automne qui attire, un week-end par an, près de 20.000 personnes autour d'une production maraîchère locale.

12. L'organisation du FitDays est gérée par une société privée dont le principal partenaire est la Mutuelle générale de l'Éducation nationale (MGEN).

\section{RÉSUMÉS}

Ce texte montre comment une institution patrimoniale peut participer à l'interprétation du territoire et à l'éducation au développement durable. Il interroge, notamment, la notion d'appropriation territoriale dans le champ de l'éducation aux territoires en contexte muséal, mettant ainsi en lumière la complexité des dynamiques de construction identitaire et de développement local.

This text shows how a heritage institution can participate in the interpretation of the territory and in education for sustainable development. In particular, it questions the notion of territorial appropriation in the field of education to territories in a museum context, thus highlighting the complexity of the dynamics of identity construction and local development.

\section{INDEX}

Mots-clés : éducation aux territoires, château, musée, centre d'interprétation, patrimoine, identité, ressource, éducation au développement durable.

Keywords : territorial education, castle, museum, interpretation centre, heritage, identity, resource, education for sustainable development.

\section{AUTEUR}

\section{OLIVIER ROUSSEAU}

Directeur du château de Bouthéon, Olivier Rousseau est attaché de conservation du patrimoine pour la ville d'Andrézieux-Bouthéon. Il est titulaire d'un doctorat en muséologie du Muséum national d'Histoire naturelle, sa thèse était consacrée à la dimension stratégique du processus muséal. 